\title{
The challenge how to improve post dialysis arterio venous (Avf) puncture sites haemostasis: is mozaik@ device new solution?
}

\begin{abstract}
Prolonged Post dialysis bleeding (PPB) of fistula needling sites is a frequent problem, which increases the hemorrhagic risk, deteriorates the quality of life of hemodialysis patients and represents a risk of blood spurts for the care givers. The increasingly ageing polyvascular dialyzed patients, with proximal brachial AVF have raised tendency to bleed. ${ }^{1}$ Anti platelets and oral anti vitamin $\mathrm{K}$ dependent anticoagulants are often prescribed increasing the risk of bleeding. Also heparin infusion during the dialysis session exacerbate prolonged post dialysis bleeding (PPB). ${ }^{2}$ The state of hydration of the dialyzed patients who are often in overload status thins the blood made it more hypocoagulable. Oral antivitamine $\mathrm{K}$ dependent anticoagulants are the largest providers of prolonged post dialysis bleeding because they fluidize blood more than the anti-platelets. ${ }^{3}$ Skin status also plays an important role, because cutaneous ageing allows the appearance of wrinkles by rarefaction of the cornea layer and by disappearance of the dermic elastin fibers promotes weak closure of the channel crossed by the needle after its removal. ${ }^{3}$
\end{abstract}

Volume 5 Issue 4 - 2017

\author{
Mokhtar Chawki \\ Department of Nephrology, Nephrokit Innovation
}

Correspondence: Mokhtar Chawki, Department of Nephrology, Owner of Nephrokit Innovation, Tel 336|444|260, Email mokhtar.chawki@orange.fr

Received: July 30, 2017 | Published: October 30, 2017

\section{Few published articles}

Usually haemostasis is achieved by removing the needles one at a time and by applying mild digital ( 2 fingers) localized direct pressure over the needle site at least 10 minutes. Absorptive haemostatic material could be simple gauze or clotting agents (e.g. Tip stop, gel foam, calcium alginate). Clotting devices e.g. tourniquets, rigid pressure clamps or straps to assist clotting are also used in nonautonomous patients. ${ }^{4}$ The danger in using these clotting agents is that the hole through the skin may be clotted but the hole into the vascular access may still be bleeding. ${ }^{4}$ The use of clotting devices masks this underlying problem. Clotting devices (as Tip stop, Clamps, braclets) exposes to the risk of vascular access thrombosis particularly in graft AVFs. They favorize puncture site stenosis on native AVFs .5 . The majority of hemodialysis literature advises against the application of excessive pressure as it may lead to iatrogenic fistula thrombosis. It is difficult to establish the right degree and duration of manual pressure. ${ }^{6,7}$

Manufacturers of haemostatic pads did not provide any published relevant evidence of the in vivo biological effect of their devices. Alginates are supposed to promote substitution between the blood sodium and calcium which is part of the alginates. To activate this biological cascade fibrin mechanism, a certain quantity of blood must be allowed to pass from the surface hole to the impregnated haemostatic pad, this blood contact is very difficult to control in high flow AVF without risk of accidental spurts! Also arterio-venous fistula is very particular kind of vessel with high blood flow that keeps the primary clot falling down raising risk of Rebleeding. Few studies have been published on the means to improve the bleeding time of AVF puncture sites, a prospective study of the chitosan* dressing of Hemcon* (chitin impregnated pad which is an adhesive molecule obtained from shrimp shell) is supposed to favor the clot by the effect of glue and also by aggregation of the red blood cells because of the positive ionic charge of chitin which agglutinates the negatively charged red blood cells. ${ }^{8}$ This study involved 15 patients who were monitored over two-weeks period to evaluate the clot at AVF puncture sites at 2 and 4 minutes and for cases greater than 4 minutes on the
Need to add a tourniquet in addition to get the final clot. The authors concluded that there was no significant difference between the conventional gauze and the Chitosan compress at 2 minutes, a slight difference at 4 minutes and a clear difference in favor of chitosan when they needs to add the tourniquet after 4 minutes.

\section{New era for compressive devices}

During 2010 Nephrokit (French start-up) patented IRISC device (Figure 1) which is a transparent microperforated adhesive acting) only by mechanical effects, allowing continuous visual control of the puncture site, so the compression is not sustained more than time to clot. This device acts also as protective screen against blood spurts. Prospective study. ${ }^{9}$ In a multicentric group of 64 patients during 3 weeks period Evaluation of post-puncture bleeding time of arterio venous fistulas with Iris* demonstrated significant superiority to stop bleeding at 3 minutes in comparison with conventional gauze ( $80 \%$ pts with Iris stops bleeding versus conventional gauze $44 \%$ pts) Figure 2. Thanks to the transparency of the device it is easy to observe direct clot achievement that allows M CHAWKI to establish some guidelines to reduce the time to clot.

\section{Mozaïk device}

During 2016 Nephrokit patented a new device Mozaik (Figure 3) transparent adhesive patch surrounded by foam frame to reduce blood leak at needle removal. Mozaiik dressing is a new compressive microperforated adhesive transparent device, surrounded by foam to increase the tightness around the puncture site after pulling off the needle. This device is tailored to fit with AVF anatomy. Advantages of the device are (Figure 4):

i. Thanks to the foam frame surrounding transparent microperforated adhesive the puncture site is perfectly sealed which prevents blood leak from the post needling hole when the needle is pulled off.

ii. The glue of the adhesive allows a reduction in the diameter of the skin puncture hole directing the blood towards the micro 
perforations while splitting its high pressure in that micropores Erythrocytes agglutinate by trapping themselves in the tortuosity of the micropores facilitating the clot formation.

iii. Inside the micropores the friction of the blood column at the periphery creates a surface tension that promotes the transformation of a continuous blood column into a drip system which drastically reduces the velocity

iv. Absorption of the excess of plasmatic fluid by the gauze placed over the microperforated adhesive will progressively enhance the thickness of blood until the clot is achieved.

v. Only Mozaïk allows blood cells passage across the channel towards the micropores without risk of spurts when the gauze is removed, erythrocytes and coagulation factors are continuously delivered to the micropores. Other clotting pads or devices do not allow this.

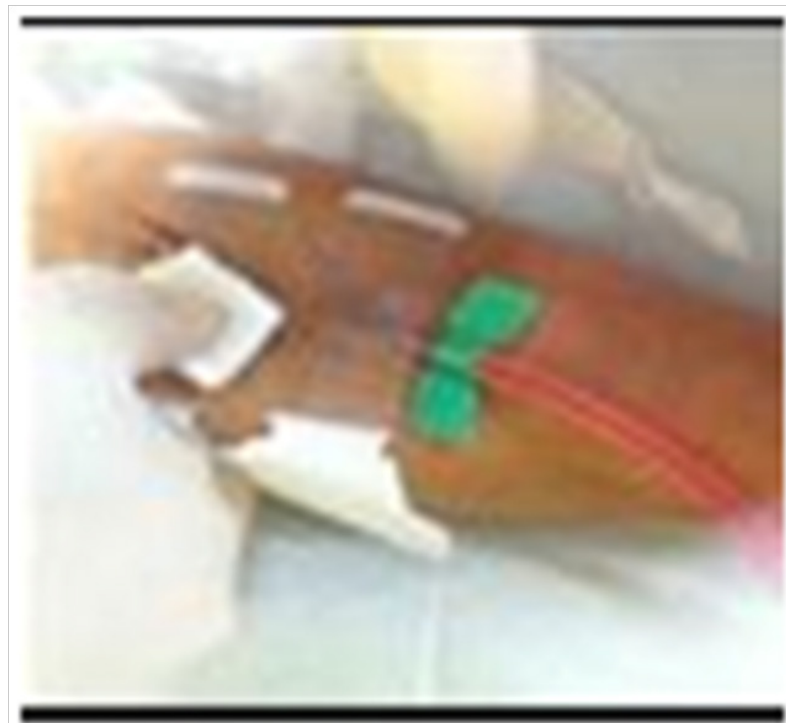

Figure I Iris@ Device.

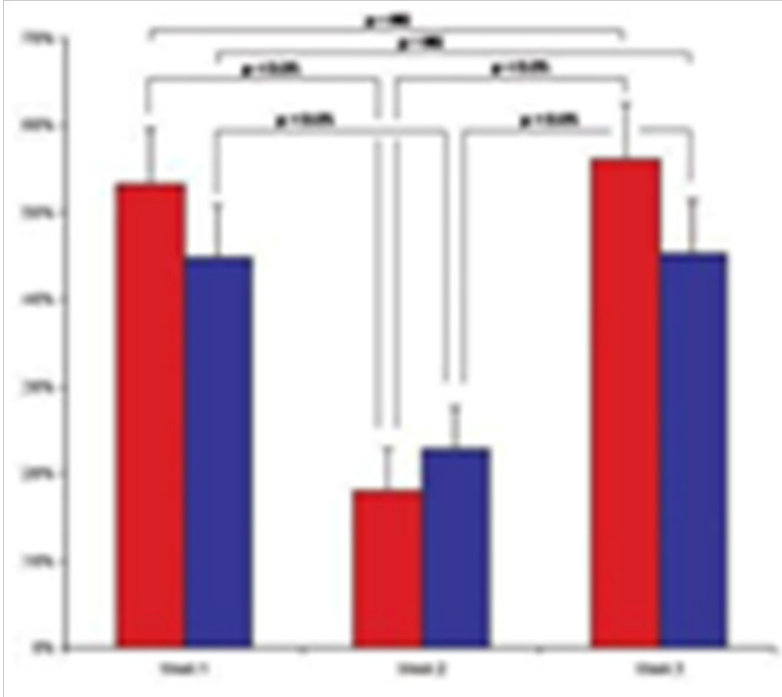

Figure 2 Clot at 3 minutes in $80 \%$ of patients versus $46 \%$ with classic gauze.

Possibility because it needs high pressure compression that reduces the flow which in turn prevents the incoming of coagulation factors to the puncture wound from the fistula. Mozaiik is the only device authorizes clot to be achieved from the vein puncture wound to the skin including the channel. The majority of published studies on AVF bleeding are only devoted to the study of compression time, but it appears that the re-bleeding of the AVF before the patient's discharge is very common with conventional devices and haemostatic dressings. Re-bleeding at the time to leave the dialysis facility is feared, but rarely reported in published studies. In recent non published prospective study (accepted abstract) (Figures $5 \& 6$ ). Guerraoui A. ${ }^{10}$ demonstrated superiority of Mozaîk to reduce by 3 the time to clot versus conventional gauze and by half the time to clot versus calcium alginate. He also found high significant difference of re bleeding episodes that happen in $33 \%$ of the patients with calcium alginate, $15 \%$ with conventional gauze and only 5\% for Mozaïk (Figure 5). Moreover in this study authors reported high frequency of Re-bleeding episodes with Calcium alginate in comparison with Gauze or Mozaïk (Figure 6).

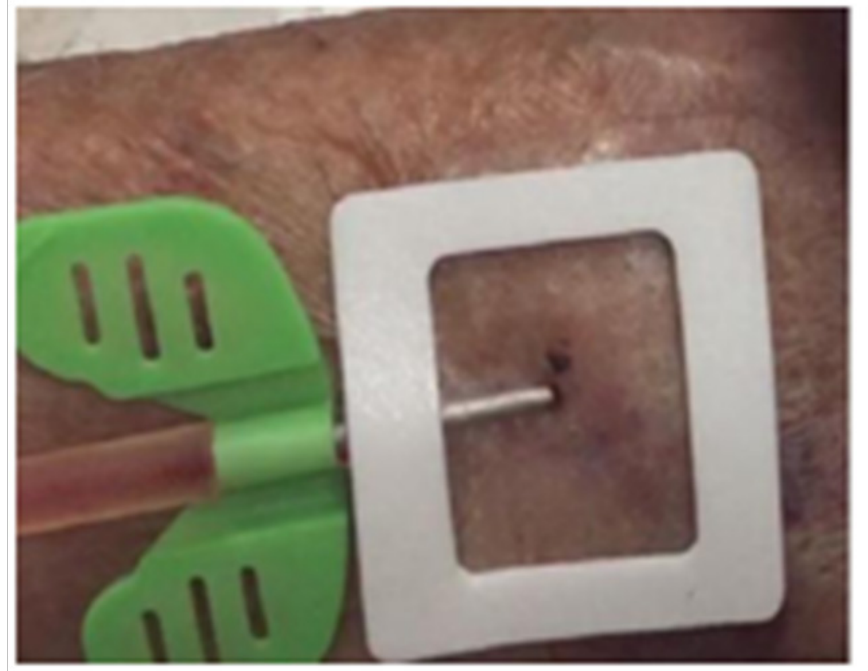

Figure 3 Mozaik @ in Place.

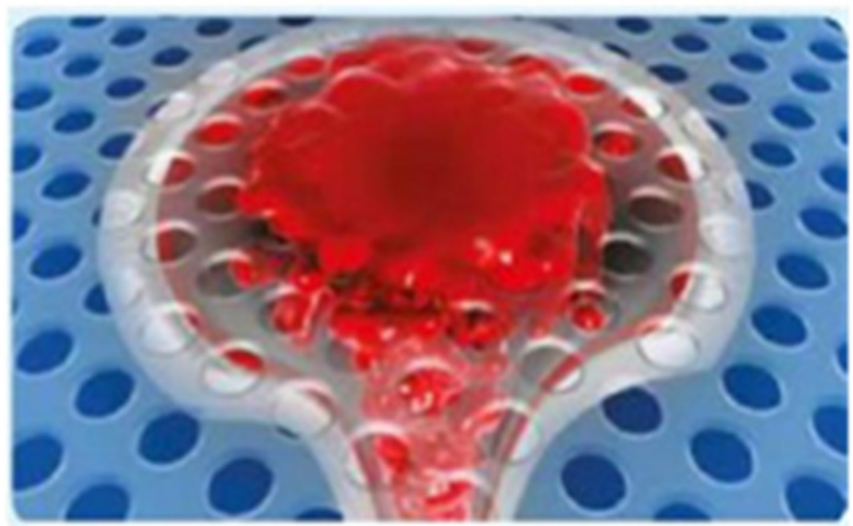

Figure 4 Mechanisms of Mozaik.

New approach to solve prolonged bleeding issue

\section{Improving diagnosis of puncture site prolonged bleeding with mozaiik@}

Consider Avf Anatomy As Combination of 3 Layers (Figure 7) One can consider the AVF as MULTILAYER visco-elastic complex where 
at the basement blood viscosity in the vessel plays a crucial role in coagulation by shear stress of the blood column circulating according rheological rules. The shear stress varies according to the number of red blood cells and the diameter of the AVF tube, viscosity is reduced in case of hyperhydratation or anaemia. Some narrowing areas (stenosis) are characterized by turbulences along the vessel. Above this deep floor there is a second viscoelastic complex represented by the sub cutaneous area, whose flexibility depends on collagenous fibers (particularly elastine fibers which became rare with ageing) this floor plays an important role at post needling step for the closure of the channel crossed by the needle through the skin. The upper floor represented by the skin surface which will become deformed at the puncture sites by aneurysms or thinning by skin ageing changes which reduces the ability of surface closure of the skin hole left by the needle.

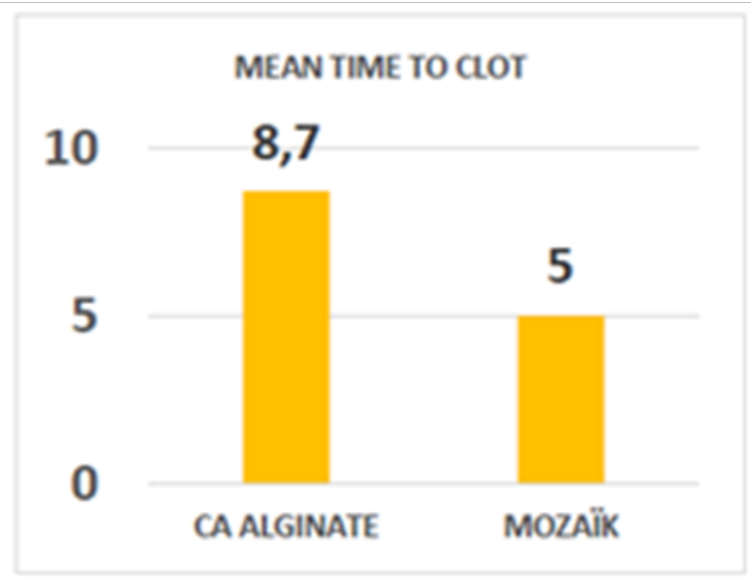

Figure 5 Mean time to clot Mozaik VS Ca Alginate.

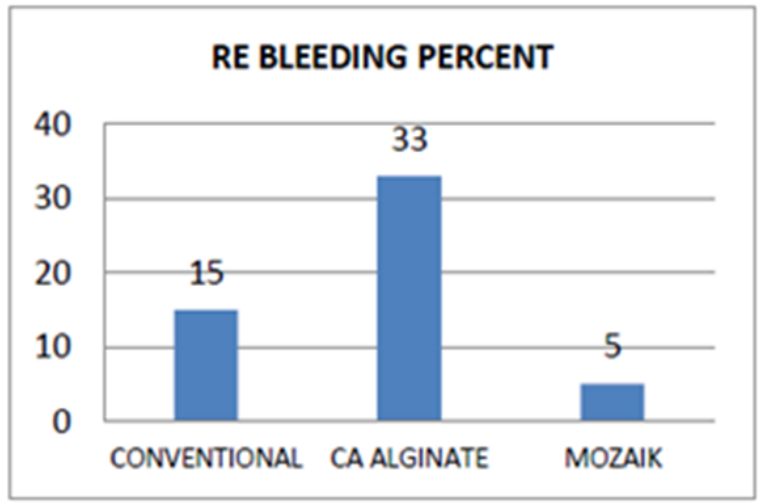

Figure 6 Rebleeding Mozaik VS Ca Alginate.

The needle will create disruption in this stratified complex and on the wall of the AVF. As long as the needle is in place the frangible joint is materially filled by the needle itself and does not bleed. During the removal of the needle, chaotic forces begins in the virtual needle tunnel that cannot be filled without obtaining a clot which needs to be in place from the venous wall to the skin. Clinical experience reported by Dr M CHAWKI the inventor of Mozaïk allows comprehensive assessment of different situations of post dialysis puncture sites delayed bleeding. In the next figures dotted lines represents Mozaik microperforated device, red cells and plasma surrounding blood cells (Figure 8)

a. In the case of normal bleeding duration (Figure 8A) the blood drop builds up in the micropores is almost spherical and limited to a single micro-pore. b. In case of hyperhydratation (Figure 8B) lighter red blood is likely more fluid and sometimes could peel off the adhesive pad crossing towards many micropores.

c. In case of anticoagulants over dosage (Figure 8C) (especially anti $\mathrm{K}$ vitamin) high fluidity of lightly dark blood raises the blood crossing many micropores

d. In obese patients (diabetics) (Figure 8D) with subcutaneous fat excess the channel of needle passage let crossing through the adhesive micropores fresh blood mixed with blood more coagulated giving a clustered appearance to the surface of the Mozaik (C), in these cases once the clot obtained we notice the presence of a clear imprint on the transparent adhesive left by the Mass of blood having passed through the transparent microperforated adhesive.

e. In case of venous stenosis (Figure 8e) as the cephalic stenosis (even stented or stenosis of the central veins) the overpressure is transmitted to the puncture hole and a spurting is observed through the micro-pores.

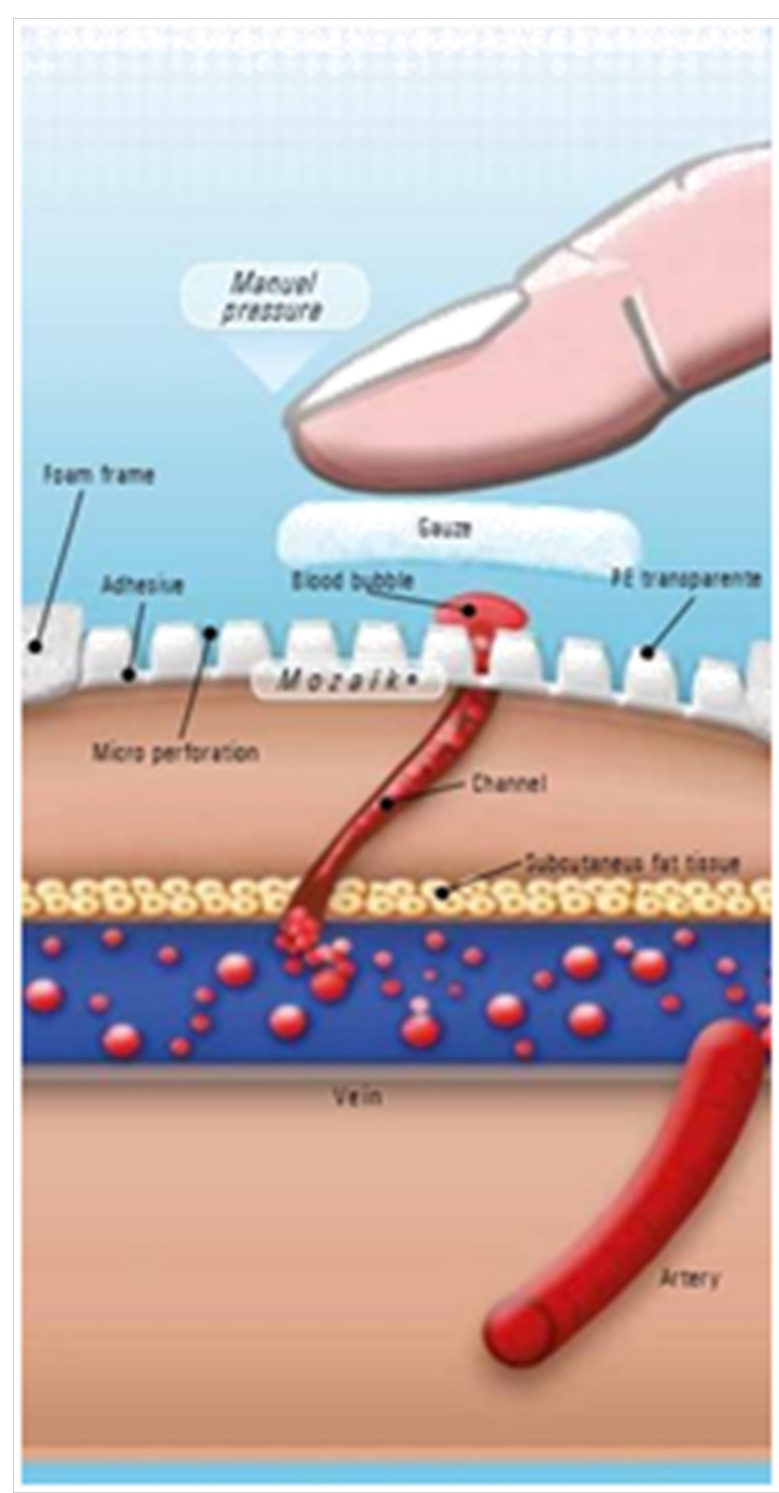

Figure 7 Puncture site anatomy. 


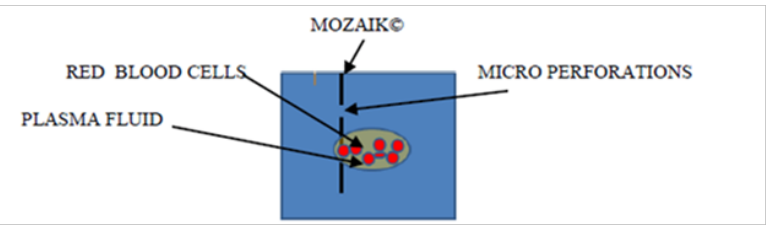

Figure 8 Mozaik microperforated device, red cells and plasma surrounding blood cells.

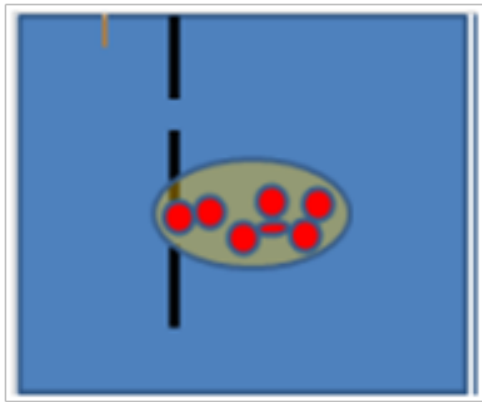

Figure 8A Normal bleeding.

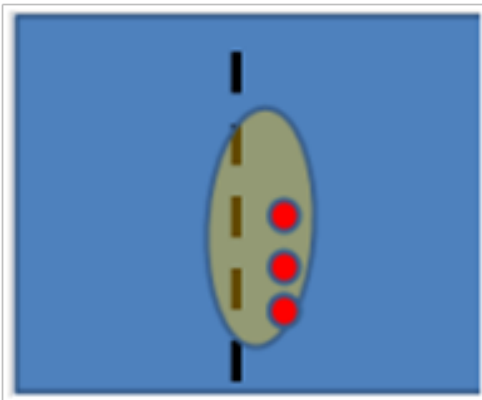

Figure 8B Hyperhydratation.

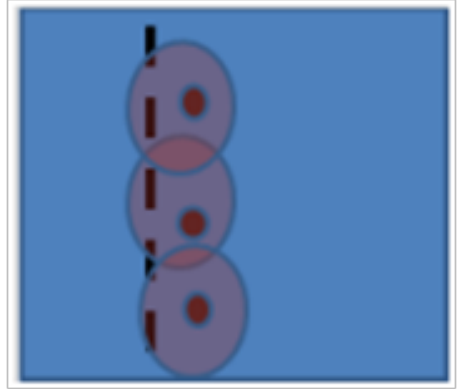

Figure 8C Cover dosage.

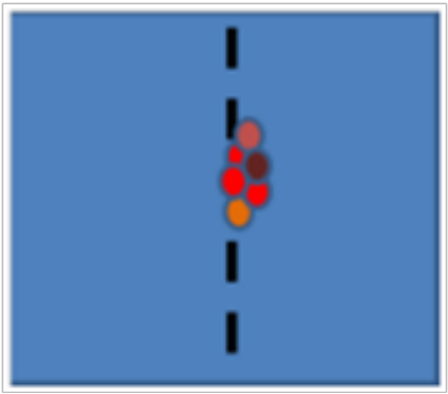

Figure 8D Clustered aspect.

In the case of post-anastomotique stenosis, or when arrhythmia occurs (Figure 8f) the blood spurt is less strong, whereas the bleeding is jerky and re-bleeding is more frequent.

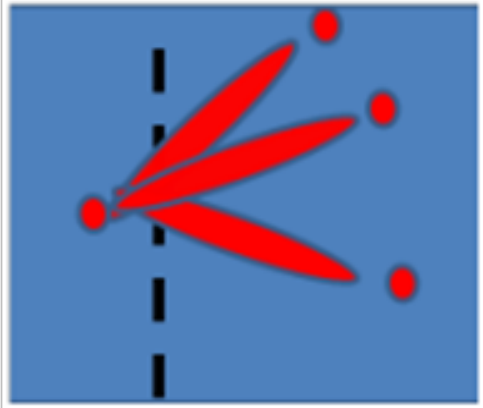

Figure 8E Central vein stenosis.

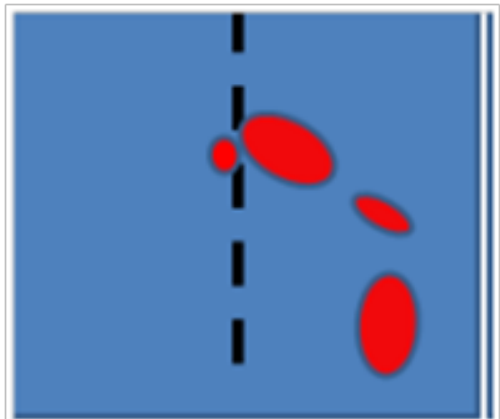

Figure 8F Post anastomotic stenosis or arrhythmia.

\section{Acknowledgements}

This work has been made possible through ISN funded fellowship.

\section{Conflicts of interest}

No financial interest or any conflict of interest exists.

\section{Funding}

None.

\section{References}

1. Galbusera M, Remuzzi G, Boccardo P. Treatment of bleeding in dialysis patient. Semin Dial. 2009;22(3):279-286.

2. Saeed F. Blood Loss through AVF: International and Al Newspaper off Nephrology Flight ID350870. 2011. p. 6.

3. M Chawki. Guidelines to reduce post dialysis bleeding. EDTNA Strasbourg. 2012.

4. Care of Needling Sites Post-Hemodialysis for fistulas and grafts (Hemostasis). BC Provincial renal Agency. 2015.

5. Simon E. The Dialysis Patient: Managing Fistula Complications in the Emergency Department. Em DOCS.net. 2016.

6. United States Renal Data System (USRDS) Annual Data Report. Epidemiology of kidney disease in the United States. 2016.

7. Hodde L, Sandroni S. Emergency department evaluation and management of dialysis patient complications. J Emerg Med. 1992;10:317-334.

8. Treatment of dialysis access puncture wound bleeding with Chitosan Dressings (NB Bachtell T Goodel and Dialysis and Transplantation. 2006. p. 1-6.

9. Boulanger. Journal of Vascular Access. 2014.

10. Guerraoui A. Prospective Study: evaluation of arterio venous fistula (AVF) compression with new device Mozaïk Patch (C) in two French hemodialysis facilities. Submited abstract Société Française de néphrologie (NICE City France). 2017. 